\title{
Acute psychosis as a harbinger of a tumor: A case of anti-N-methyl-D-aspartate receptor encephalitis
}

\author{
Tyler J Gleason, Anthony J Donato, Dilli R Poudel \\ Internal Medicine, Reading Health System, West Reading, PA, USA
}

Received: February 14, 2017

Accepted: March 7, 2017

Online Published: March 9, 2017

DOI: $10.5430 /$ crim.v4n2p25

URL: https://doi.org/10.5430/crim.v4n2p25

\begin{abstract}
An obese but otherwise healthy 22-year-old female presented to the hospital with an episode of acute psychosis. On exam the patient was febrile and had labile systolic blood pressure. Urine drug screen and infectious workup were negative. A CSF Anti-N-methyl-D-aspartate (Anti-NMDA) receptor antibody was positive. Brain magnetic resonance imaging (MRI) showed hyperintensity in the left hippocampus on diffusion weighted imaging (DWI). The patient was started on prednisone, intravenous immunoglobulins, and rituximab. Hospital course was complicated by seizures and autonomic instability, requiring prolonged stay in the ICU. Cognitive, motor, and autonomic function gradually improved with therapy. Magnetic resonance imaging of the pelvis revealed a $3 \mathrm{~cm} \times 3 \mathrm{~cm}$ left ovarian teratoma that was removed with oophorectomy. The patient was discharged to rehab hospital for continued treatment and physical therapy.
\end{abstract}

Key Words: Psychosis, Encephalitis, Autoimmune, Paraneoplastic, Teratoma

\section{INTRODUCTION}

Anti-N-methyl-D-aspartate (Anti-NMDA) receptor encephalitis is an uncommonly described entity with the first case-series of 12 patients reported in 2007 and less than 1,000 total cases described in the literature worldwide. ${ }^{[1,2]}$ Nonetheless, it is increasingly being recognized as the underlying cause in many cases of idiopathic encephalitis and acute psychosis. The diagnosis is made difficult not only by the novelty of the disease and rarity of cases, but also by the diffuse constellation of symptoms with which patients may present. However, the majority of cases are seen in young females and share certain clinical features, including a non-specific, viral-like prodrome, prominent psychiatric symptoms, cognitive dysfunction, seizures and/or seizurelike dyskinesias, and autonomic instability. Anti-NMDA receptor encephalitis is associated with benign ovarian ter- atomas in roughly $50 \%$ of patients, and removal of these tumors improves clinical outcomes. Physicians should consider this disorder in patients with acute psychosis and cognitive impairment, especially in young females, who account for roughly $80 \%$ of cases. ${ }^{[2]}$

\section{Case presentation}

A morbidly obese 22-year-old female without prior psychiatric history or substance abuse was brought by her mother to the emergency room with a chief complaint of altered mental status. Her symptoms began four days before presentation with severe headaches and bouts of anxiety and short-term memory loss. The headaches were initially attributed to her menstrual cycle, as she had complained of similar perimenstrual symptoms in the past. However, her mother became more concerned after having an extended conversation with the patient, who left the room and then returned five minutes

*Correspondence: Tyler J Gleason; Email: Tyler.Gleason@readinghealth.org; Address: 420 S. 5th Avenue, West Reading, PA 19611, USA. 
later with no recollection of their previous conversation. She was not on any medications and had no known allergies. She denied any recent travel, bites, rash, or sexual activity. She also denied any tobacco, alcohol, or illicit drug use. There was no family history of mental health disorders.

On exam, she was febrile to $38.0^{\circ} \mathrm{C}$ with labile blood pressures ranging from 110 to 190 systolic. She was fearful and hesitant to answer questions, though fully oriented to person, place, and time. When asked to count backwards from 100 by 7's, the patient's entire demeanor changed instantly and dramatically. She became violent, striking members of the staff and claiming that she was trapped in a bad dream. She required physical restraints and chemical sedation for the safety of herself and others. Even with large doses of chemical sedation, she experienced a waxing and waning level of consciousness, with periods of relative calm punctuated by episodes of violent thrashing and severe agitation. By the next day, she became convinced she was a character on the television program The Walking Dead. She described the show's plot and characters as ongoing events of her life.

On initial laboratory workup, urine drug screen was negative and urinalysis was normal. Brain computed tomography (CT) was within normal limits. Blood cultures were drawn and lumbar puncture (LP) was performed. Cerebrospinal fluid (CSF) studies showed 130 white blood cells (WBC) with $96 \%$ lymphocytes, 0 red blood cells, mildly elevated glucose, and normal protein. Brain MRI showed hyperintensity in the left hippocampus on diffusion weighted imaging. Intravenous (IV) acyclovir was begun empirically to cover herpes simplex virus (HSV) encephalitis. She was also started on IV vancomycin and ceftriaxone, although bacterial infection was thought to be less likely. Consults were placed to infectious disease and neurology. Infectious disease recommended continuation of IV acyclovir, CSF testing for various viral encephalitides, and discontinuation of antibiotics if blood cultures were negative after 48 hours. Neurology evaluated the patient and also felt that this was most likely a viral meningitis picture.

Over the next five days, the patient continued to have a waxing/waning level of consciousness, with periods of relative calm, punctuated by episodes of severe agitation. CSF bacterial and fungal cultures, gram stains, and viral assays were all negative. CSF autoantibody studies revealed antiNMDA receptor antibodies with a titer of 1:640. Antibiotics and acyclovir were discontinued and the patient was started on prednisone, intravenous immunoglobulins (IVIG), and rituximab. Hospital course was complicated by repeated seizures and severe autonomic instability, characterized by periods lasting 20-30 seconds with heart rate as low as 10 beats/minute, requiring transfer to the intensive care unit and dopamine infusions. However, her seizures and autonomic instability improved and eventually resolved after two weeks of immunotherapy. Her cognitive function also gradually improved and her delusional thinking subsided. Ultrasound of the pelvis was performed and revealed a $2.9 \mathrm{~cm} \times 2.9 \mathrm{~cm}$ cyst that was poorly visualized due to body habitus. MRI of pelvis was performed and revealed a $3.9 \mathrm{~cm} \times 3.9 \mathrm{~cm} \times$ $3.8 \mathrm{~cm}$ benign mature cystic teratoma at the anterior margin of the right ovary. She underwent right abdominal salpingoophorectomy without complications. Several days later, she was transferred to a rehabilitation facility for continued physical and cognitive therapy. The patient spent a total of 1 month in the hospital. She returned home after two weeks in the rehabilitation hospital, at which time her cognitive functioning had improved markedly, but had not yet returned to baseline. She also experienced significant improvements in her strength and motor functioning and was able to ambulate 150 feet without assistance. Formal neuropsychological testing was scheduled for the following month to assess for any residual cognitive deficits.

\section{Discussion}

Initial differential diagnosis for a previously healthy young female presenting with acute psychosis includes druginduced psychosis, a focal brain lesion, encephalitis (both viral and autoimmune), and a primary psychiatric condition. In a patient with suspected encephalitis, workup should include lumbar puncture, with viral and autoimmune CSF studies, electroencephalogram (EEG), and MRI of the brain. ${ }^{[3]}$ CSF studies will typically show lymphocytosis with few oligoclonal bands, no red blood cells, and normal glucose and protein levels. MRI abnormalities are present in $23 \%$ $50 \%$ of cases and typically show non-specific enhancement in the cortex or subcortical structures, although diffusion tensor imaging (DTI) may reveal widespread white matter damage. ${ }^{[4]}$ EEG shows non-specific generalized slowing in up to $90 \%$ of cases. ${ }^{[3]}$ Brain biopsy has been done in some patients, but typically shows normal tissue or nonspecific changes, therefore is generally not indicated. Serum anti-NMDA receptor antibodies are present in only $15 \%$ of cases. $^{[3]}$ Definitive diagnosis is made by the detection of antiNMDA receptor antibodies in the CSF, which should prompt pelvic ultrasound (US) and MRI to evaluate for presence of teratoma, which is found in up to $50 \%$ of females older than 18 with this disorder. ${ }^{[5]}$

Clinical course can be positively altered with immunosuppressants and removal of teratomas, which are frequently associated with the disorder, however physicians must have a high index of suspicion so that they order appropriate serol- 
ogy to make the diagnosis. First-line therapy includes corticosteroids, IVIG, or plasmapheresis. Rituximab is a promising second-line agent that can be added if initial therapy fails or if patient symptoms are particularly severe. Cyclophosphamide can also be used as an adjunctive agent, although is less preferred due to toxicity concerns. Prompt removal of teratomas, when found, may obviate the need for secondary agents, such as rituximab and cyclophosphamide. Full recovery can take up to 2 years, with symptoms often abating in the inverse order of their development. Early diagnosis and treatment with immunotherapy improves outcomes and short- ens the recovery period. ${ }^{[6]}$ Approximately $75 \%$ of patients either fully recover or are left with only mild deficits, while the remaining $25 \%$ die or are left with severe deficits. The risk of relapse is $20 \%-25 \%$ and can occur months to years after the initial presentation and can sometimes be provoked by tapering of immunotherapy. ${ }^{[6]}$ Therefore, these patients need close long-term follow-up and care should be taken in the tapering of immunotherapy.

\section{Conflicts OF InTEREST Disclosure}

The authors have no competing interests to declare.

\section{REFERENCES}

[1] Dalmau J, Tüzün E, Wu HY, et al. Paraneoplastic anti-N-methyl-Daspartate receptor encephalitis associated with ovarian teratoma. Ann Neurol. 2007; 61(1): 25-36. PMid:17262855 https://doi.org/ 10.1002/ana.21050

[2] Barry H, Byrne S, Barrett E, et al. Anti-N-methyl-d-aspartate receptor encephalitis: review of clinical presentation, diagnosis and treatment. BJPsych Bull. 2015; 39(1): 19-23. Available from: https : //www.ncbi.nlm.nih.gov/pubmed/?term=26191419

[3] Mann AP, Grebenciucova E, Lukas RV. Anti-N-methyl-D-aspartatereceptor encephalitis: Diagnosis, optimal management, and challenges. Therapeutics and Clinical Risk Management. 2014; 10: 51724. PMid:25061311 https://doi .org/10.2147/TCRM.S61967
[4] Heine J, Prüss H, Bartsch T, et al. Imaging of autoimmune encephalitis - Relevance for clinical practice and hippocampal function. Neuroscience. 2015; 309: 68-83. PMid:26012492 https: //doi.org/10.1016/j.neuroscience.2015.05.037

[5] Florance NR, Davis RL, Lam C, et al. Anti-N-methyl-D-aspartate receptor (NMDAR) encephalitis in children and adolescents. Ann Neurol. 2009; 66(1): 11-8. PMid:19670433 https://doi.org/10 .1002/ana. 21756

[6] Dalmau J, Lancaster E, Martinez-Hernandez E, et al. Clinical experience and laboratory investigations in patients with anti-NMDAR encephalitis. The Lancet Neurology. 2011; 10: 63-74. https : //doi.org/10.1016/S1474-4422(10)70253-2 